\title{
Hepatitis B virus Reactivation in Patients Receiving Tocilizumab for Rheumatoid Arthritis: A Long-term, Retrospective Observational Study
}

\section{Kuo Meng Hsuan}

Dalin Tzu Chi Hospital https://orcid.org/0000-0003-3124-3104

Chih-Wei Tseng ( $\nabla$ cwtseng@gmail.com )

https://orcid.org/0000-0002-6951-4646

Ming-Chi Lu

Dalin Tzu Chi Hospital

Chien-Hsueh Tung

Dalin Tzu Chi Hospital

Kuo-Chih Tseng

Dalin Tzu Chi Hospital

Kuang-Yung Huang

Dalin Tzu Chi Hospital

Chi-Hui Lee

Dalin Tzu Chi Hospital

Ning-Sheng Lai

Dalin Tzu Chi Hospital

\section{Research article}

Keywords: HBV reactivation, Hepatitis flare, Rheumatoid Arthritis, Tocilizumab

Posted Date: May 12th, 2020

DOI: https://doi.org/10.21203/rs.3.rs-27343/v1

License: (c) (i) This work is licensed under a Creative Commons Attribution 4.0 International License. Read Full License 


\section{Abstract}

Aim To investigate the risk of hepatitis B virus (HBV) reactivation in patients undergoing long-term tocilizumab (TCZ) therapy for rheumatoid arthritis (RA).

Method From January 2011 through August 2019, a total of 134 RA patients who received TCZ at Dalin Tzu Chi Hospital were screened. Patients were excluded if they were $<20$ years, without complete data, or received TCZ for less than 3 months. A total of 97 patients were enrolled in this retrospective study. Clinical data, co-medications, and the occurrence of HBV reactivation were recorded.

Results Of the 97 enrolled patients, 7 were HBsAg+ (7.2\%), 64 were HBsAg-/HBcAb+ (61\%) and 26 were $\mathrm{HBsAg}-/ \mathrm{HBcAb}+(26.8 \%)$. The median disease follow-up time was 9 years (range, 1-18 years). TCZ was administered for a median of 29 months (range, 3-91 months). Four patients (4.1\%) experienced HBV reactivation after TCZ therapy. Of the $7 \mathrm{HBsAg}+$ patients, 4 received antiviral prophylaxis and had no HBV reactivation; the remaining 3 patients had no antiviral prophylaxis, and all 3 (100\%) experienced early HBV reactivation and hepatitis flare (median time to event, 6 months; range, 5-8 months). Hyper-bilirubinemia occurred in 2 of these 3 patients, with mild prothrombin time prolongation in one. After salvage entecavir treatment, all patients had a favorable outcome. Of the $64 \mathrm{HBsAg}-/ \mathrm{HBcAb}+$ patients, only one became positive for serum HBV DNA $(2.5 \times 107 \mathrm{IU} / \mathrm{mL})$ after 18 months of TCZ treatment $(1.6 \% ; 1 / 64)$. This patient was immediately treated with entecavir, which prevented hepatitis flare.

Conclusions HBsAg+ RA patients undergoing TCZ treatment are at high risk of HBV reactivation, which is prevented by antiviral prophylaxis. $\mathrm{HBsAg}-/ \mathrm{HBcAb}+$ patients also are at risk of $\mathrm{HBV}$ reactivation. Although their risk of reactivation is lower than that of $\mathrm{HBsAg}+$ patients, strict monitoring of their HBV status is still necessary.

\section{Background}

The risk for hepatitis B virus (HBV) reactivation during immunosuppressive therapy in patients with chronic or occult HBV infection is increasingly recognized in different disciplines of medicine, including rheumatology. ${ }^{1,2}$ The consequences of HBV reactivation during immunosuppressive therapy range from an asymptomatic increase in serum HBV DNA levels to fatal hepatic failure. ${ }^{3}$ In rheumatoid arthritis (RA), HBV can be reactivated by changes in the disease course and by drug use. ${ }^{4-7}$ Inflammatory arthritis patients receiving biologic disease-modifying antirheumatic drugs (bDMARDs) are thought to have a moderate risk (1-10\%) of hepatitis $\mathrm{B}$ reactivation. ${ }^{7} \mathrm{~A}$ previous study reports that without antiviral prophylaxis, HBV reactivation occurs in $62.5 \%$ of HBV carriers and $25 \%$ of patients with occult HBV infection undergoing treatment with tumor necrosis factor -alpha blockers. ${ }^{8}$

Tocilizumab (TCZ), a humanized monoclonal antibody that selectively neutralizes both the soluble and membranebound forms of interleukin (IL)-6 receptor, is a recently developed biological agent that effectively alleviates RA disease symptoms. IL-6 is a cytokine that plays an important role in regulating immune response and inflammation. ${ }^{9}$ Both the soluble and membrane-bound forms of the IL- 6 receptor ${ }^{10}$ can induce stress pathways and promote the pathogenesis of autoimmunity. ${ }^{11}$ Current treatment guidelines suggest that patients with HBV surface antigen-positive $(\mathrm{HBsAg}+)$ should initiate antiviral prophylaxis before immunosuppressive or cytotoxic therapy. $7,12,13$ However, determining the risk of TCZ-induced HBV reactivation is difficult due to a lack of prospective controlled studies. Several studies have investigated the risk of HBV reactivation in HBsAg+ patients receiving TCZ. ${ }^{14-16}$ Although the data are limited, the risk of HBV reactivation in these patients is as high as $60 \%$ 
$(3 / 5)^{14}$ and may require liver transplantation for hepatic failure. ${ }^{16}$ Further evidence shows that HBV reactivation does not occur in HBsAg+ patients receiving anti-HBV prophylaxis. ${ }^{14,15}$ Most studies report that HBV reactivation does not occur in $\mathrm{HBsAg}-/ \mathrm{HBV}$ core antibody-positive $(\mathrm{HBcAb}+)$ patients, ${ }^{14,17-19}$ although $\mathrm{HBV}$ reactivation with DNA reappearance was observed in Japanese studies of a small number of patients ${ }^{20-22}$

Because the number of studies investigating HBV reactivation in RA patients treated with TCZ is limited, the optimal management protocol is still unclear. Therefore, the aim of this retrospective, real-world study is to determine the risk of HBV reactivation with long-term TCZ therapy for RA patients in our hospital.

\section{Methods}

\section{Patients}

We retrospectively reviewed the medical records of RA patients who had received TCZ therapy between January 2011 to August 2019 at Dalin Tzu Chi General Hospital. The inclusion criteria were as follows: (1) fulfilled the RA classification criteria; ${ }^{23,24}$ (2) available HBsAg and HBcAb status at diagnosis; and (3) treated with TCZ for at least 3 months. Patients were excluded if they were $<20$ years of age or lacked required data. In accordance with international guidelines, TCZ was administered at the standard intravenous dose of 4-- $8 \mathrm{mg} / \mathrm{kg}$ every 4 weeks or subcutaneous dosage of 162 mg every $1-2$ weeks. ${ }^{25}$

\section{Follow up of study population}

Medical records were reviewed retrospectively. Baseline characteristics included serum markers of HBV (HBsAg, HBcAb, hepatitis B surface antibody [HBsAb], and HBV DNA), anti-hepatitis C virus antibody (anti-HCV), liver biochemical parameters (serum aspartate aminotransferase [AST]; alanine aminotransferase [ALT]), comorbidity, co-medications, and the occurrence of HBV reactivation. Patients were monitored for ALT/AST levels every 3 months, and HBsAg and HBV DNA tests were checked whenever clinically indicated. Detailed medical records were collected, including immunological profiles, TCZ course, previous and concomitant DMARD therapy, and corticosteroid therapy.

\section{Assessment of HBV reactivation and hepatitis flare}

The primary endpoint of this study was the $\mathrm{HBV}$ reactivation rate. For $\mathrm{HBsAg}(+)$ patients, $\mathrm{HBV}$ reactivation was defined as an increase in HBV DNA > $2 \log _{10}$ over that of baseline or an absolute level of HBVDNA $>4 \log _{10}$ if baseline data were unavailable. ${ }^{12} \mathrm{~A}$ change in HBV DNA titer from negative to positive or HBsAg seroreversion (HBsAg reappearance) was defined in HBsAg (-)/HBcAb (+). ${ }^{12}$ Hepatitis flare was defined as an ALT level elevated at least 3 times that of baseline and $>100 \mathrm{U} / \mathrm{L}^{12}$

\section{Statistical analysis}

The data were analyzed using SPSS 19.0 for Windows (SPSS Inc, Chicago, IL, USA). Categorical variables are presented as counts and percentages, and continuous variables are presented as the median value and range.

\section{Results}

\section{General characteristics of the study subjects}


The enrolled patient distribution is illustrated in Figure 1. Of the initial 134 RA patients receiving TCZ, 97 remained after exclusions for missing data regarding HBsAg or HBcAb status $(n=25)$ or receiving TCZ for less than 3 months $(n=12)$. Of the included 97 patients, 7 were $\mathrm{HBsAg}+(7 / 97,7.2 \%)$ and 90 were $\mathrm{HBsAg}-(90 / 97,92.8 \%)$. Of the HBsAg- patients, 64 were HBsAg-/HBcAb+ (64/90, 71.1\%) and 26 were HBsAg-/HBcAb- (26/90, 28.9\%). Four patients $(4 / 99,4.1 \%)$ experienced HBV reactivation during TCZ treatment.

The clinical, serological, and virologic characteristics and therapeutic regimens of the patient cohort are shown Table 1. The median age was 64 years (range, 38-87), and 75 (77\%) were female. The median disease follow-up time was 9 years (range, 1-18 years). TCZ was administered for a median of 29 months (range, 3-91) in combination with methotrexate (71 patients, $73 \%$ ) or a low dose of glucocorticoids (90 patients, $94 \%$ ). Seventy-one (73\%) patients had a history of other bDMARD treatment. Most patients had previously received adalimumab (45 patients; $46 \%$ ) or rituximab (25 patients; $26 \%)$.

\section{Clinical Features of HBsAg+ patients with HBV reactivation}

The general characteristics of the HBsAg+ patients are shown in Table 1. The median age of these patients was 65 years (range, 56-78 years). They received TCZ with median 16 months (range, 4-66 months). Before using TCZ, patients were previously treated with methotrexate (MTX), glucocorticoids, and at least one type of bDMARDs (Table 1). Adalimumab (3 patients; 43\%) and rituximab (3 patients; 43\%) were the most common bDMARDs.

Of the $7 \mathrm{HBsAg}+$ patients, 4 (57.1\%) received antiviral prophylaxis and none experienced HBV reactivation or hepatitis flare. In contrast, HBV reactivation and hepatitis flare were noted in all 3 patients without antiviral prophylaxis (Figure 1). Clinical data for the patients with HBV reactivation are shown in Table 2. All were female (median age, 77 years; range, 56-78 years), and the median time to reactivation from the initiation of TCZ treatment was 6 months (range, 5-8 months). Hyperbilirubinemia was noted in 2 of the 3 patients, and mildly prolonged prothrombin time was observed in one patient. No acute liver decompensation (i.e., ascites, hepatic encephalopathy, hepatorenal syndrome, or variceal bleeding) was reported. All 3 of these patients received the antiviral agent entecavir, which achieved sustained viral suppression and smooth recovery with no complications. The time course of HBV reactivation is described below and shown in Figure $2 A-C$.

\section{Case 1}

A 56-year-old female who was diagnosed with RA in 2006 was treated with adalimumab from June 2011 to Aug 2012. Because her response to this agent was poor, she started TCZ in Sep 2012. In Jun 2013 (after 5 months of TCZ therapy), routine blood tests showed elevated ALT (155 U/L) and a high viral load $\left(3.7 \times 10^{7} \mathrm{IU} / \mathrm{mL}\right)$. Under the diagnosis of HBV reactivation, entecavir ( $0.5 \mathrm{mg} /$ day) was initiated (Figure 2A). After 2 months of antiviral treatment, the HBV viral load had decreased to $301 \mathrm{lU} / \mathrm{mL}$ and the ALT level had normalized. TCZ treatment was continued later, and no further adverse events were noted.

\section{Case2}

A 77-year-old-female who was diagnosed with RA in 2004 was treated with etanercept for 5 months and rituximab for 20 months. Because her response was insufficient, she began TCZ in June 2012. In Dec 2012 (after 6 months of TCZ treatment), elevated ALT (698 U/L) and a high viral load $\left(1.6 \times 10^{7} \mathrm{IU} / \mathrm{mL}\right)$ were noted (Figure 2B). Entecavir ( $0.5 \mathrm{mg} /$ day was initiated under the diagnosis of HBV reactivation. Hyperbilirubinemia (maximum, $24.8 \mathrm{mg} / \mathrm{dL}$ ) and abnormal ALT level (maximum, $946 \mathrm{U} / \mathrm{mL}$ ) persisted for 4 weeks. Prolonged prothrombin time (INR, 1.81) was also 
observed. The ALT returned to within the normal range about 9 weeks later, and the jaundice improved 23 weeks later. TCZ use was discontinued.

\section{Case 3}

A 78-year-old-female who was diagnosed with RA in 2003 was treated with etanercept for 46 doses starting in Jan 2007 and then switched to rituximab for 13 months. TCZ was initiated in October 2012. Eight months after the first TCZ, she was sent to our ER due to a urinary tract infection. Routine laboratory tests revealed a slightly elevated ALT level (106 U/L) with an HBV viral load of $1.7 \times 10^{8} \mathrm{IU} / \mathrm{mL}$ (Figure $2 \mathrm{C}$ ). Entecavir ( $0.5 \mathrm{mg} /$ day) was initiated and TCZ administration was stopped. Hyperbilirubinemia (maximum, $4.3 \mathrm{mg} / \mathrm{dL}$ ) and abnormal ALT level (maximum, 355 $\mathrm{IU} / \mathrm{mL}$ ) persisted for 20 weeks. Thirty-four weeks later, the HBV viral load was undetectable and ALT had returned to within the normal range. TCZ was no longer used.

\section{Clinical Features of $\mathrm{HBsAg}-/ \mathrm{HBcAb}+$ patients with $\mathrm{HBV}$ reactivation}

The general characteristics of the $\mathrm{HBsAg}-/ \mathrm{HBcAb}+$ patients are shown in Table 1 . The median age of patients was 67 years (range, 38-87 years). They received TCZ treatment for a median time of 26 months (range, 3-84 months). The most common co-medications were methotrexate (47 patients; 73\%) and low- to medium-dose glucocorticoids (64 patients, 100\%). Forty-five (70\%) patients had a history of other bDMARD treatment. Most patients had previously received adalimumab (31 patients; 48\%). With TCZ administration, one HBsAg-/HBcAb+ patient (1/64; $1.6 \%$ ) experienced HBV reactivation (Figure 1; Table 2). The time course of HBV reactivation is described below and shown in Figure 2D.

\section{Case 4}

A 67-year-old-female, diagnosed with RA in 2003, had received etanercept for 35 months, adalimumab for 25 months, and rituximab for 25 months. The last dose of bDMARDs was administrated 17 months before she started TCZ (March 2013). After 18 months of TCZ treatment (Oct 2014,) HBsAg seroreversion with serum HBV DNA appearance $\left(2.5 \times 10^{7} \mathrm{IU} / \mathrm{mL}\right)$ was observed. Her serum ALT remained stable without evidence of hepatitis flare (Figure 2D). Entecavir (0.5 mg/day) was initiated, and the HBV viral load was undetectable 22 months later. TCZ treatment was continued.

\section{Discussion}

Our results show that RA patients receiving TCZ are at risk of HBV reactivation. The risk was very high for HBsAg+ patients who were not administered an antiviral prophylactic agent $(3 / 3 ; 100 \%)$. This risk was eliminated by antiviral prophylaxis. HBV reactivation in $\mathrm{HBsAg}+$ patients often occurred within the first year of TCZ treatment (median administration time, 6 months; range, 5-8 months) and was associated with hepatitis flare. We also observed one case of HBV reactivation among HBsAg-/HBcAb+ patients $(1 / 64 ; 1.6 \%)$.

Patients treated with TCZ are at risk for serious infections, including tuberculosis and other opportunistic bacterial, invasive fungal, and viral infections. The European Society of Clinical Microbiology and Infectious Diseases suggests that TCZ use puts patients at modest risk of overall infection. ${ }^{13}$ Because patients with HBV infection were excluded from pivotal TCZ trials, ${ }^{26,27}$ the risk of HBV reactivation was not well reported. Several studies discuss the clinical presentation and risk of HBV reactivation in HBsAg+ patients in the absence of antiviral prophylaxis (Supplement Table 1). ${ }^{14,16}$ A prospective study of $5 \mathrm{HBsAg}+\mathrm{RA}$ patients receiving TCZ without antiviral prophylaxis 
reports an HBV reactivation rate of $60 \%(3 / 5) .{ }^{14}$ Together with our observation that all $3 \mathrm{HBsAg}+$ patients without antivirus prophylaxis experienced HBV reactivation, these data indicate a high HBV reactivation rate among HBsAg+ patients taking TCZ and stress the importance of antiviral prophylaxis in these patients.

The early reactivation of HBV observed in our HBsAg+ patients during TCZ therapy is consistent with other reports (Supplement material Table 1). ${ }^{14,16}$ A case study from the Netherlands reports that one HBsAg+ patient experienced HBV reactivation with fulminant hepatitis 2 weeks after a single dose of TCZ. ${ }^{16}$ Although antiviral treatment was immediately administered, hepatic failure ensued, requiring liver transplantation. ${ }^{16} \mathrm{~A}$ prospective study from China reports that HBV reactivation occurred 1-3 months after patients received 3 doses of TCZ, with none experiencing hepatitis flare. ${ }^{14}$ After antiviral treatment, all of these patients recovered well. In our study, the median time to reactivation from the first TCZ dose was 6 months (range, 5-8 months). All patients with HBV reactivation developed hepatitis flare up, and 2 even had hyperbilirubinemia (2/3;67\%). After entecavir treatment, we observed improvements in liver function (median time to improvement, 4 months; range, 1-5 months) and hyperbilirubinemia (median time to improvement, 5 months; range, 3-6 months). These reports indicate a short interval between the first dose of TCZ and HBV reactivation (Supplemental Table 1) The reactivation may result in fulminant hepatitis despite early preemptive treatment.

In contrast to the high rate of HBV reactivation in $\mathrm{HBsAg}+$ patients without antiviral prophylaxis, none of the $\mathrm{HBsAg}+$ patients who received antiviral prophylaxis experienced HBV reactivation in our study (Figure 1). A similar result was reported in a retrospective study from Taiwan, with no HBV reactivation occurring in $11 \mathrm{HBsAg}+\mathrm{RA}$ patients receiving TCZ with antiviral prophylaxis. ${ }^{15}$ For HBsAg+ RA patients receiving TCZ, close monitoring is not enough. Due to the high risk of HBV reactivation, short interval between TCZ administration and reactivation, and possibility of fulminant hepatitis, our results indicate that antiviral prophylactic agents should be given to HBsAg+ patients before TCZ therapy.

The reported rates of HBV reactivation among $\mathrm{HBsAg}-/ \mathrm{HBcAb}+$ patients under TCZ treatment are inconsistent. Five studies including a total of 120 patients revealed no HBV reactivation events. ${ }^{14,17-19,28}$ Most of those studies included patients taking multiple types of bDMARDs and only a small number taking TCZ (range, 7-30 patients). ${ }^{17-}$ 19, 28 A prospective study in China investigating the short-term safety of TCZ (3-dose treatment and 3 months of observation $)^{14}$ reported no HBV reactivation in $\mathrm{HBsAg}-/ \mathrm{HBCAb}+$ patients $(\mathrm{n}=41)$. Three Japanese studies ${ }^{20,21,29}$ reported total 4 cases of HBV reactivation among HBsAg-/HBcAb+ RA patients receiving TCZ (Supplement material Table 2). In these small cohorts (range, 4-25 patients), the HBV reactivation rates were 4-25\%. ${ }^{20,21,29}$ The viral load in 3 of the 4 cases of HBV reactivation fluctuated between undetectable to detectable, and all cases resolved spontaneously without antiviral treatment. Those results are limited by the small case number and short monitoring time but suggest that TCZ treatment is safe for $\mathrm{HBsAg}-/ \mathrm{HBCAb}+$ patients.

Our cohort included $64 \mathrm{HBsAg}-/ \mathrm{HBCAb}+$ patients who received TCZ for a median time of 26 months (range, 3-84 months). Of these patients, only one experienced HBsAg seroreversion with a high HBV viral load $\left(2.5 \times 10^{7} \mathrm{IU} / \mathrm{mL}\right)$; thus, the $\mathrm{HBV}$ reactivation rate was $1.6 \%$ (1/64) among $\mathrm{HBsAg}-/ \mathrm{HBcAb}+\mathrm{RA}$ patients. This result indicates that $\mathrm{HBsAg}-/ \mathrm{HBcAb}+\mathrm{RA}$ patients receiving TCZ are at risk for HBV reactivation. The monitoring of HBV-DNA and HBV seromarkers every 3-6 months can identify HBV reactivation at an earlier stage and should be suggested in this population. 
This study has several limitations. First, the retrospective, observational design precludes the control of numerous factors, and some data are missing. However, our investigation includes the largest cohort of any study of this subject. Because of the large number of person-years of follow up, our findings nonetheless provide important information regarding treatment decisions for prophylaxis and monitoring in this population. Second, our study only enrolled Asian patients; whether our findings are applicable to other populations is uncertain.

\section{Conclusion}

RA patients receiving TCZ therapy should be assessed for risk of HBV reactivation using HBV seromarkers. While this risk is very high in HBsAg+ patients, HBV reactivation can be prevented by the administration of antiviral prophylactic agents. For HBsAg-/HBcAb+ patients, the risk of HBV reactivation is lower but still present. We recommend the monitoring of HBV-DNA and HBV markers in this patient population.

\section{Abbreviations}

ALT, alanine aminotransferase;

AST, aspartate aminotransferase;

HBV, hepatitis B virus;

HBsAg, HBV surface antigen;

HBcAb, HBV core antibody;

HBsAb, HBV surface antibody;

HCV, hepatitis C virus;

IL, interleukin;

RA, rheumatoid arthritis;

sDMARDs, synthetic disease-modifying antirheumatic drugs;

bDMARDs, biological disease-modifying antirheumatic drugs;

TCZ, tocilizumab;

T. bil, total bilirubin

\section{Declarations}

\section{Ethics approval and consent to participate}

The study conformed to the ethical guidelines of the 1975 Declaration of Helsinki as reflected by a priori approval by the Ethics Committee of Dalin Tzu Chi General Hospital (approval number B10901013). The requirement for informed consent was waived because this study was retrospective in nature and the data analyzed did not include patient identification. 


\section{Consent for publication}

Not applicable.

\section{Availability of data and materials}

Please contact the author for data requests.

\section{Competing interests}

The authors declare no financial and non-financial interests.

\section{Funding}

This study was funded by the Dalin Tzuchi General Hospital through grant number DTCRD109-T-34.

\section{Author contributions}

Meng Hsuan Kuo: material support, drafting of the manuscript

Chih-Wei Tseng: statistical analysis, material support, critical revision of the manuscript for important intellectual content

Ming-Chi Lu: critical revision of the manuscript for important intellectual content

Chien-Hsueh Tung: critical revision of the manuscript for important intellectual content

Kuo-Chih Tseng: material support, critical revision of the manuscript for important intellectual content

Kuang-Yung Huang: critical revision of the manuscript for important intellectual content

Chi-Hui Lee: critical revision of the manuscript for important intellectual content

Ning-Sheng Lai: material support

\section{Acknowledgements}

Not applicable.

\section{References}

1. Lok AS, Ward JW, Perrillo RP, et al. Reactivation of hepatitis B during immunosuppressive therapy: potentially fatal yet preventable. Annals of internal medicine 2012;156:743.

2. Vassilopoulos D, Calabrese LH. Management of rheumatic disease with comorbid HBV or HCV infection. Nature Reviews Rheumatology 2012;8:348.

3. Di Bisceglie AM, Lok AS, Martin P, et al. Recent US Food and Drug Administration warnings on hepatitis B reactivation with immune-suppressing and anticancer drugs: just the tip of the iceberg? Hepatology 2015;61:703-711.

4. Jansen TL, Mulder C. Rheumatology meets hepatology in 2012: a clinician's guideline for TNF inhibitors in hepatitis B/C virus carriers. Expert Opinion on Biological Therapy 2012;12:391-393. 
5. Kuo MH, Tseng C-W, Lee C-H, et al. Moderate Risk of Hepatitis B Virus Reactivation in HBsAg-/HBcAb+ carriers Receiving Rituximab for Rheumatoid Arthritis. Scientific Reports 2020;10:1-9.

6. Chen Y-M, Yang S-S, Chen D-Y. Risk-stratified management strategies for HBV reactivation in RA patients receiving biological and targeted therapy: a narrative review. Journal of Microbiology, Immunology and Infection 2019;52:1-8.

7. Reddy KR, Beavers KL, Hammond SP, et al. American Gastroenterological Association Institute guideline on the prevention and treatment of hepatitis $B$ virus reactivation during immunosuppressive drug therapy. 2015;148:215-219.

8. Lan J-L, Chen Y-M, Hsieh T-Y, et al. Kinetics of viral loads and risk of hepatitis B virus reactivation in hepatitis B core antibody-positive rheumatoid arthritis patients undergoing anti-tumour necrosis factor alpha therapy. Annals of the rheumatic diseases 2011;70:1719-1725.

9. Nishimoto N, Kishimoto T. Interleukin 6: from bench to bedside. Nature Reviews Rheumatology 2006;2:619.

10. Liu X, Jones GW, Choy EH, et al. The biology behind interleukin-6 targeted interventions. Current opinion in rheumatology 2016;28:152-160.

11. Calabrese LH, Rose-John S. IL-6 biology: implications for clinical targeting in rheumatic disease. Nature Reviews Rheumatology 2014;10:720.

12. Terrault NA, Lok AS, McMahon BJ, et al. Update on prevention, diagnosis, and treatment of chronic hepatitis B: AASLD 2018 hepatitis B guidance. Hepatology 2018;67:1560-1599.

13. Winthrop KL, Mariette X, Silva JT, et al. ESCMID Study Group for Infections in Compromised Hosts (ESGICH) Consensus Document on the safety of targeted and biological therapies: an infectious diseases perspective (Soluble immune effector molecules [II]: agents targeting interleukins, immunoglobulins and complement factors). Clinical Microbiology and Infection 2018;24:S21-S40.

14. Chen LF, Mo YQ, Jing J, et al. Short-course tocilizumab increases risk of hepatitis $B$ virus reactivation in patients with rheumatoid arthritis: a prospective clinical observation. 2017;20:859-869.

15. Lin C-T, Huang W-N, Hsieh C-W, et al. Safety and effectiveness of tocilizumab in treating patients with rheumatoid arthritis-A three-year study in Taiwan. Journal of Microbiology, Immunology and Infection 2019;52:141-150.

16. Sonneveld MJ, Murad SD, van der Eijk A, et al. Fulminant Liver Failure due to Hepatitis B Reactivation During Treatment With Tocilizumab. ACG Case Reports Journal 2019;6:e00243.

17. Papalopoulos I, Fanouriakis A, Kougkas N, et al. Liver safety of non-tumour necrosis factor inhibitors in rheumatic patients with past hepatitis B virus infection: an observational, controlled, long-term study. Clin Exp Rheumatol 2018;36:102-109.

18. Barone M, Notarnicola A, Lopalco G, et al. Safety of long-term biologic therapy in rheumatologic patients with a previously resolved hepatitis B viral infection. Hepatology 2015;62:40-46.

19. Ahn SS, Jung SM, Song JJ, et al. Safety of Tocilizumab in Rheumatoid Arthritis Patients with Resolved Hepatitis B Virus Infection: Data from Real-World Experience. 2018;59:452-456.

20. Nakamura J, Nagashima T, Nagatani K, et al. Reactivation of hepatitis B virus in rheumatoid arthritis patients treated with biological disease-modifying antirheumatic drugs. International Journal of Rheumatic Diseases 2016;19:470-475.

21. Urata $Y$, Uesato $R$, Tanaka $D$, et al. Prevalence of reactivation of hepatitis $B$ virus replication in rheumatoid arthritis patients. Modern Rheumatology 2011;21:16-23. 
22. Watanabe T, Fukae J, Fukaya S, et al. Incidence and risk factors for reactivation from resolved hepatitis B virus in rheumatoid arthritis patients treated with biological disease-modifying antirheumatic drugs. International Journal of Rheumatic Diseases 2018;22:574-582.

23. Arnett FC, Edworthy SM, Bloch DA, et al. The American Rheumatism Association 1987 revised criteria for the classification of rheumatoid arthritis. Arthritis \& Rheumatism 1988;31:315-324.

24. Aletaha D, Neogi T, Silman AJ, et al. 2010 rheumatoid arthritis classification criteria: an American College of Rheumatology/European League Against Rheumatism collaborative initiative. Arthritis \& Rheumatism 2010;62:2569-2581.

25. Smolen JS, Landewe R, Bijlsma J, et al. EULAR recommendations for the management of rheumatoid arthritis with synthetic and biological disease-modifying antirheumatic drugs: 2016 update. Ann Rheum Dis 2017;76:960-977.

26. Emery $\mathrm{P}$, Keystone $\mathrm{E}$, Tony $\mathrm{H}$, et al. IL-6 receptor inhibition with tocilizumab improves treatment outcomes in patients with rheumatoid arthritis refractory to anti-tumour necrosis factor biologicals: results from a 24-week multicentre randomised placebo-controlled trial. Annals of the rheumatic diseases 2008;67:1516-1523.

27. Burmester GR, Rubbert-Roth A, Cantagrel A, et al. A randomised, double-blind, parallel-group study of the safety and efficacy of subcutaneous tocilizumab versus intravenous tocilizumab in combination with traditional disease-modifying antirheumatic drugs in patients with moderate to severe rheumatoid arthritis (SUMMACTA study). Annals of the rheumatic diseases 2014;73:69-74.

28. Carlino G, Fornaro M, Santo L, et al. Occult HBV infection may negatively impact on drug survival in patients with rheumatoid arthritis on treatment with a first biologic drug. An appraisal from the Biologic Apulian Registry (BIOPURE). Reumatismo 2019;71:24-30.

29. Watanabe T, Fukae J, Fukaya S, et al. Incidence and risk factors for reactivation from resolved hepatitis B virus in rheumatoid arthritis patients treated with biological disease-modifying antirheumatic drugs. 2019;22:574582.

\section{Tables}

Table 1. Patient baseline characteristics. 
All $(\mathrm{N}=97)$ HBsAg+ $(\mathrm{N}=7) \mathrm{HBsAg}-/ \mathrm{HBc} \mathrm{Ab}+(\mathrm{N}=64)$

Age (years) ${ }^{\#}$

64 (38-87) 65 (56-78) 67 (38-87)

Sex, Female n (\%)

$75(77 \%) \quad 5(71 \%)$

$49(77 \%)$

Follow up time (years)

$9(1-18) \quad 9(2-14)$

$9(1-18)$

Duration of TCZ infusion (months) ${ }^{\#}$

Anti-HCV (+), n (\%)

$14(14 \%) \quad 1(14 \%)$

$26(3-84)$

$11(17 \%)$

Antirheumatic therapies before TCZ use, n (\%)

sDMARDs

\begin{tabular}{llll} 
Methotrexate & $91(94 \%)$ & $7(100 \%)$ & $62(97 \%)$ \\
\hline Azathioprine & $3(3 \%)$ & $0(0 \%)$ & $3(5 \%)$ \\
Cyclosporine & $15(15 \%)$ & $3(43 \%)$ & $9(14 \%)$ \\
\hline Leflunomide & $45(46 \%)$ & $3(43 \%)$ & $26(41 \%)$ \\
\hline Sulfasalazine & $85(88 \%)$ & $6(86 \%)$ & $57(89 \%)$ \\
\hline Glucocorticoid (oral)* & $96(99 \%)$ & $7(100 \%)$ & $63(98 \%)$ \\
\hline bDMARDs & $10(10 \%)$ & $1(14 \%)$ & $8(17 \%)$ \\
\hline Abatacept & $45(46 \%)$ & $3(43 \%)$ & $31(48 \%)$ \\
\hline Adalimumab & $22(23 \%)$ & $2(29 \%)$ & $11(17 \%)$ \\
\hline Etanercept & $13(13 \%)$ & $1(14 \%)$ & $8(13 \%)$ \\
\hline Golimumab & $25(26 \%)$ & $3(43 \%)$ & $12(19 \%)$ \\
\hline Rituximab & $26(27 \%)$ & $0(0 \%)$ & $19(30 \%)$ \\
\hline bDMARs naïve & & & \\
\hline
\end{tabular}

Concomitant, n (\%)

Methotrexate

Glucocorticoid (oral)*

equivalent dose (mg)

Low dose
$71(73 \%) \quad 4(57 \%) \quad 47(73 \%)$

$96(99 \%) \quad 7(100 \%) \quad 64(100 \%)$

$5(2-10) \quad 5(2.5-7.5) \quad 5(2-10)$

$90(94 \%) \quad 7(100 \%) \quad 58(91 \%)$

Page $11 / 16$ 


\begin{tabular}{llll}
\hline Moderate dose & $6(6 \%)$ & $0(0 \%)$ & $6(9 \%)$ \\
\hline Azathioprine & $1(1 \%)$ & $0(0 \%)$ & $1(2 \%)$ \\
\hline Cyclosporine & $4(4 \%)$ & $1(14 \%)$ & $3(5 \%)$ \\
\hline Leflunomide & $21(22 \%)$ & $0(0 \%)$ & $13(20 \%)$ \\
\hline Sulfasalazine & $45(47 \%)$ & $3(43 \%)$ & $28(42 \%)$ \\
\hline
\end{tabular}

\#Data are presented as the median (range)

*Glucocorticoids (oral): cortisone, prednisolone, methylprednisolone, dexamethasone Prednisolone equivalent dose (mg/day): High dose, $>20 \mathrm{mg} /$ day $\geq 4 \mathrm{wk}$; Moderate dose, 10-20 $\mathrm{mg} /$ day $\geq 4 \mathrm{wk}$; Low dose, $<10 \mathrm{mg} /$ day $\geq 4 \mathrm{wk}$ Abbreviations: HBV, hepatitis B virus; HCV, hepatitis C virus; HBsAg, HBV surface antigen; $\mathrm{HBcAb}$, HBV core antibody; TCZ, tocilizumab; bDMARDs, biological disease-modifying antirheumatic drugs; sDMARDs, synthetic disease-modifying antirheumatic drugs

Table 2. Clinical features of RA patients who developed HBV reactivation during tocilizumab therapy. 


\begin{tabular}{|c|c|c|c|c|}
\hline & & & & $\mathrm{Ab}+$ \\
\hline ata source & Case 1 & Case 2 & Case 3 & Case 4 \\
\hline ge/Sex & $56 / F$ & $77 / F$ & $78 / \mathrm{F}$ & $67 / F$ \\
\hline A & 11 & 14 & 11 & 15 \\
\hline isease(year) & & & & \\
\hline $\begin{array}{l}\text { CZ dose at } \\
\text { sactivation }\end{array}$ & 5 & 6 & 8 & 18 \\
\hline $\begin{array}{l}\text { BsAg } \\
\text { sroreversion }\end{array}$ & - & - & - & + \\
\hline $\mathrm{BsAb}$ & $\mathrm{N}$ & $\mathrm{N}$ & $\mathrm{N}$ & $\mathrm{N}$ \\
\hline nti-HCV & NA & NA & $\mathrm{N}$ & $\mathrm{N}$ \\
\hline $\begin{array}{l}\text { BV } \quad \text { DNA } \\
\text { seak, } \mathrm{IU} / \mathrm{mL} \text { ) }\end{array}$ & $3.7 \times 10^{7}$ & $1.6 \times 10^{7}$ & $1.7 \times 10^{8}$ & $2.5 \times 10^{7}$ \\
\hline $\begin{array}{l}\text { LT } \\
\text { nitial/peak, } \\
\text { /L) }\end{array}$ & $155 / 155$ & $698 / 946$ & $106 / 355$ & $98 / 107$ \\
\hline $\begin{array}{l}. \text { bil } \\
\text { nitial/peak, } \\
\text { (g/dL) }\end{array}$ & $0.6 / 1.3$ & $2.2 / 24.8$ & $1.1 / 4.3$ & $0.5 / 0.5$ \\
\hline $\mathrm{T}$ & $\mathrm{N}$ & $\mathrm{N}$ & $Y$ & $\mathrm{~N}$ \\
\hline
\end{tabular}

rolongation

scites

$\mathrm{N} \quad \mathrm{N}$

$\mathrm{N}$

$\mathrm{N}$

epatic

$\mathrm{N}$

$\mathrm{N}$

$\mathrm{N}$

$\mathrm{N}$

.cephalopathy

epatitis flare $\mathrm{Y}$

$\mathrm{Y}$

reatment for ETV $0.5 \mathrm{mg}$

ETV $0.5 \mathrm{mg}$ Y

$\mathrm{N}$

BV

sactivation

utcome

alive \& well

alive \& well

alive \& well

alive \& well

ntirheumatic therapies before TCZ

DMARDs

ADA (14)

ETN

(5)/Rituximab ETN

ETN (35)/ADA 
nonths)

sDMARDs MTX/Pd/SSZ/CSA MTX/Pd/SSZ/LEF/CSA MTX/Pd/SSZ/LEF

MTX/Pd/SSZ/LEF

oncomitant immunosuppressants MTX 0 7.5

7.5 7.5

(mg/week)

Prednisolone 5 7.5 7.5 7.5

equivalent

dose

(mg/day)

SDMARDs $\mathrm{N}$

SSZ

SSZ

SSZ

Entecavir, ETV; NAA, nucleic acid analogue; IFX, infliximab; TAC, Tacrolimus; RA, rheumatoid arthritis; HBV, hepatitis B virus; HCV, hepatitis C virus; F, female; TCZ, tocilizumab; HBsAg, HBV surface antigen; HBc Ab, antibody against HBV core antigen; ALT, alanine aminotransferase; IU, international units; T.bil, Total Bilirubin ;PT, prothrombin time; sDMARD, Pd, prednisolone; MTX, methotrexate; LEF, leflunomide; SSZ, sulfasalazine; CSA, cyclosporine, bDMADRs, ADA, adalimumab; ETN, etanercept; NA, not applicable; N, not happened; Y, happened

\section{Figures}




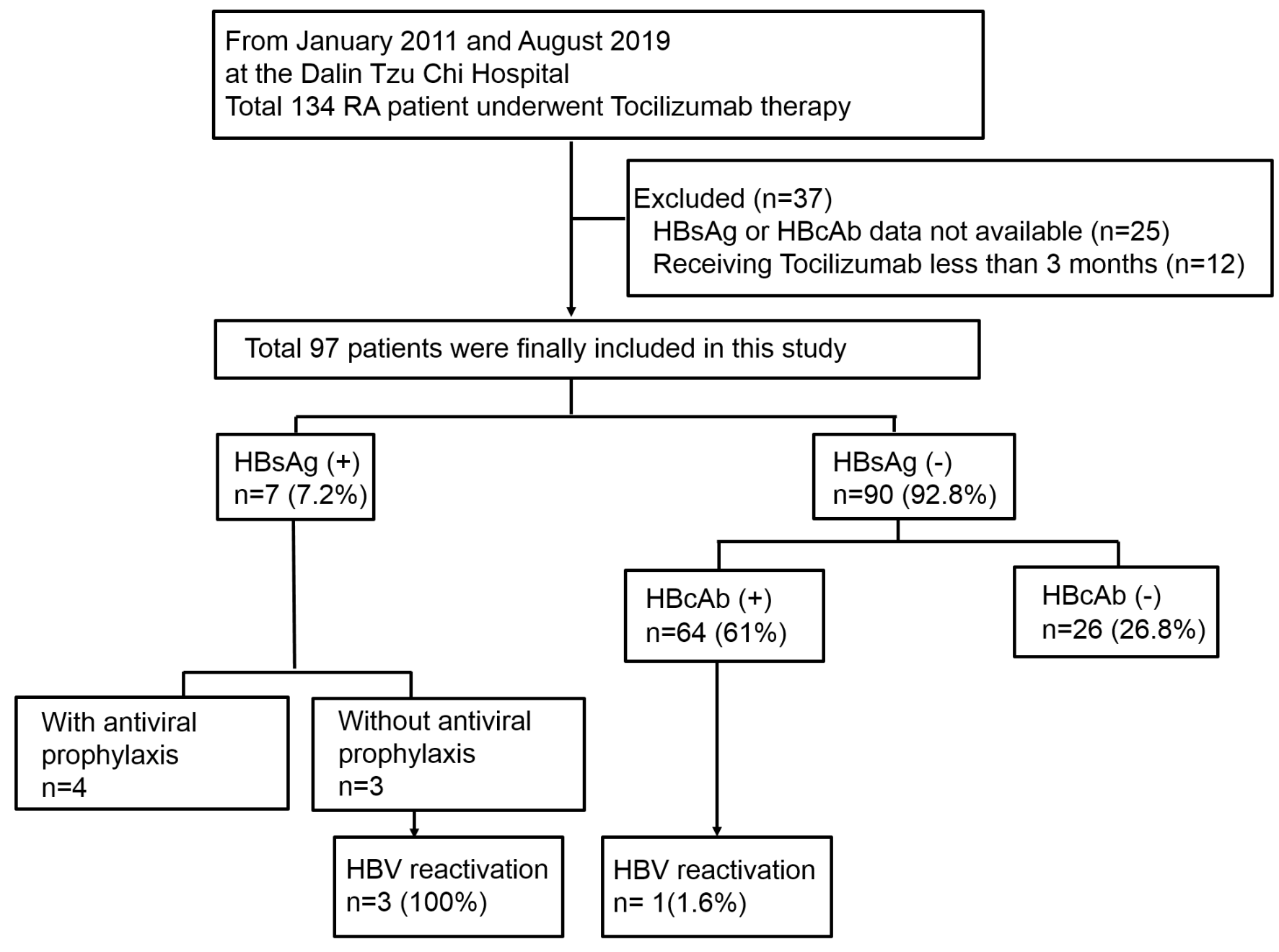

Figure 1

Flow diagram of study cohort characteristics. 2 Flowchart shows the hepatitis B virus (HBV) infection serostatus distribution at baseline and 3 the occurrence of HBV reactivation in rheumatoid arthritis (RA) patients treated with 4 tocilizumab. 5 Abbreviations: HBsAg, HBV surface antigen; HBcAb, HBV core antibody 


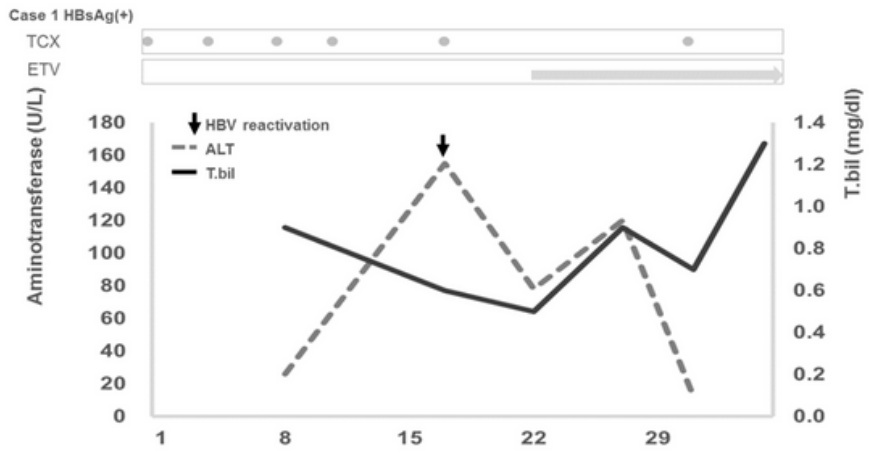

HBV DNA (IU/mL) $\uparrow_{3.7 \times 10^{7}}^{\uparrow}$

$\uparrow_{301} \quad$ Time (week)

A

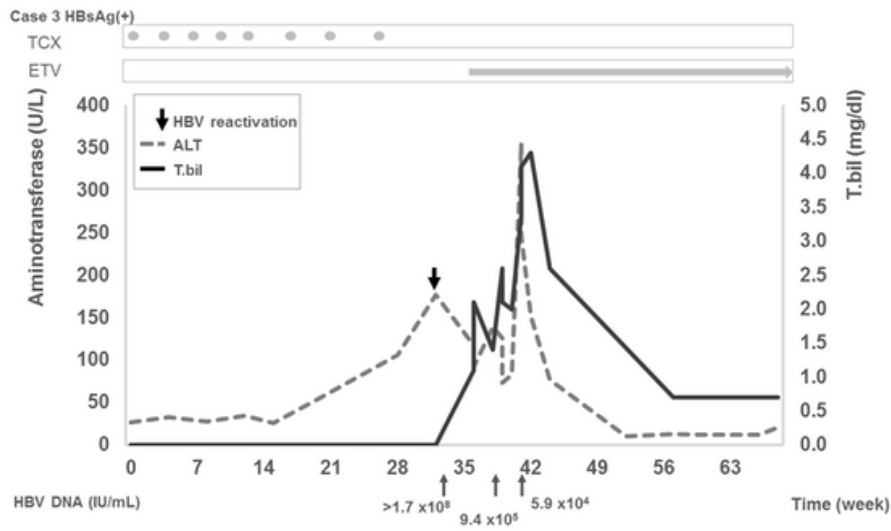

C

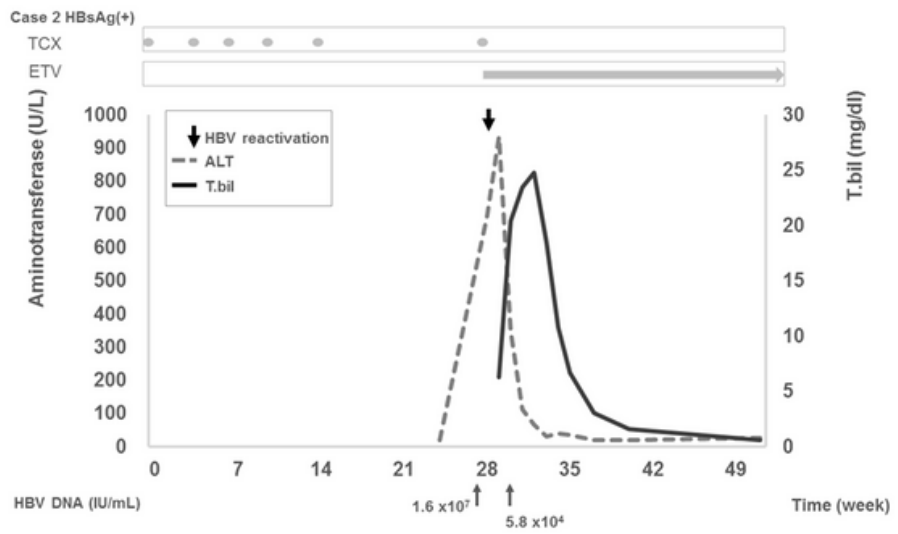

B

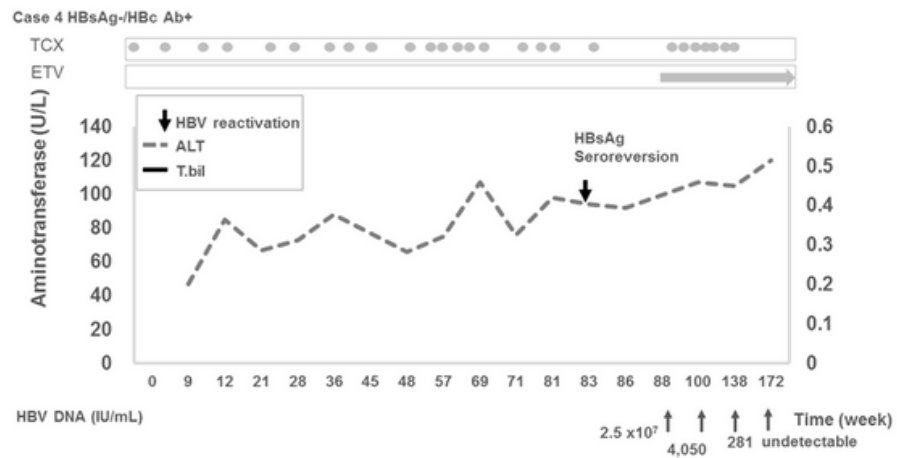

D

Figure 2

Time course of serum alanine aminotransferase and total bilirubin 1 concentrations in patients experiencing HBV reactivation. 2 Abbreviations: ALT, alanine aminotransferase; T. bil, total bilirubin; HBV, hepatitis B virus; 3 HBsAg, HBV surface antigen; HBcAb, HBV core antibody; TCZ, tocilizumab; ETV, entecavir

\section{Supplementary Files}

This is a list of supplementary files associated with this preprint. Click to download.

- LaTeXSupportingFiles.pdf 